Georgian Mathematical Journal

Volume 11 (2004), Number 1, 7-26

\title{
LINEARIZATION AND HIGHER ORDER NONLINEAR OSCILLATION THEOREMS USING COMPARISON METHODS
}

\author{
RAVI P. AGARWAL, SAID R. GRACE, AND DONAL O'REGAN
}

\begin{abstract}
We establish some new oscillation criteria for higher order nonlinear differential equations. The main idea involves comparing our equation with the related linear second order differential equations so that the known oscillation theorems from the literature can be employed directly.
\end{abstract}

2000 Mathematics Subject Classification: 34C10, 34C15.

Key words and phrases: Linearization, oscillation, nonoscillation, nonlinear, comparison.

\section{INTRODUCTION}

In this paper we shall deal with the oscillatory behavior of all solutions of the functional differential equation

$$
L_{n} x(t)+q(t) f(x[g(t)])=0,
$$

where $n \geq 2$, and

$$
\left\{\begin{array}{l}
L_{0} x(t)=x(t), \\
L_{k} x(t)=\frac{1}{a_{k}(t)} \frac{d}{d t}\left(L_{k-1} x(t)\right), \quad k=1,2, \ldots, n-1, \\
L_{n} x(t)=\frac{d}{d t}\left(\left[L_{n-1} x(t)\right]^{\alpha}\right) .
\end{array}\right.
$$

In what follows we shall assume that

(i). $a_{i}(t) \in C\left(\left[t_{0}, \infty\right), \mathbb{R}^{+}=(0, \infty)\right), t_{0} \geq 0$,

$$
\int^{\infty} a_{i}(s) d s=\infty, \quad i=1,2, \ldots, n-1,
$$

(ii). $q(t) \in C\left(\left[t_{0}, \infty\right), \mathbb{R}^{+}\right)$,

(iii). $g(t) \in C\left(\left[t_{0}, \infty\right), \mathbb{R}=(-\infty, \infty)\right)$ and $\lim _{t \rightarrow \infty} g(t)=\infty$,

(iv). $f \in C(\mathbb{R}, \mathbb{R})$ and $x f(x)>0$ for $x \neq 0$, and

(v). $\alpha$ is the quotient of positive odd integers.

The domain $\mathcal{D}\left(L_{n}\right)$ of $L_{n}$ is defined to be the set of all functions $x:\left[t_{x}, \infty\right) \rightarrow$ $\mathbb{R}, t_{x} \geq t_{0}$, such that $L_{j} x(t), j=0,1, \ldots, n$, exist and are continuous on $\left[t_{x}, \infty\right)$. Our attention is restricted to those solutions $x \in \mathcal{D}\left(L_{n}\right)$ of equation (1.1) which satisfy $\sup \{|x(t)|: t \geq T\}>0$ for every $T \geq t_{x}$. We make the standing hypothesis that equation (1.1) does possess such solutions. A solution of equation (1.1) is called oscillatory if it has arbitrarily large zeros; otherwise, 
it is called nonoscillatory. Equation (1.1) is called oscillatory if all its solutions are oscillatory.

Our interest in this paper is to relate the oscillation of the nonlinear higher order equation (1.1), when $f$ is not required to be a monotone function, to an appropriate linear second order ordinary differential equation. This enables us to employ numerous known results from the literature, see [4], [5].

In addition we establish some new oscillation criteria for higher order nonlinear equations of type (1.1). The obtained results are extended to obtain new oscillation criteria for neutral equations of the form

$$
L_{n}(x(t)+p(t) x[h(t)])+q(t) f(x[g(t)])=0,
$$

where $L_{n}$ and the functions $g, q$ and $f$ are as in equation (1.1), $p(t) \in$ $C\left(\left[t_{0}, \infty\right), \mathbb{R}^{+} \cup\{0\}\right)$ and $h(t) \in C\left(\left[t_{0}, \infty\right), \mathbb{R}\right)$ and $\lim _{t \rightarrow \infty} h(t)=\infty$.

Finally in Section 4 we consider a special case of equation (1.1), namely, the equation

$$
\frac{d}{d t}\left(x^{(n-1)}(t)\right)^{\alpha}+q(t) f(x[g(t)])=0 .
$$

We note that linearization of nonlinear oscillation theorems has been the subject of intensive study and for some interesting results, we refer to Grace [7]-[8], Kwong and Wong [9] and Philos [11]. We also remark that several other related results are available in [2], [3].

\section{Preliminaries}

To formulate our results we shall use the following notation: Let $a_{i}(t) \in$ $C\left(\left[t_{0}, \infty\right), \mathbb{R}\right), i=1,2, \ldots$, we define $I_{0}=1$,

$$
I_{i}\left(t, s ; a_{i}, a_{i-1}, \ldots, a_{1}\right)=\int_{s}^{t} a_{i}(u) I_{i-1}\left(u, s ; a_{i-1}, \ldots, a_{1}\right) d u, \quad i=1,2, \ldots
$$

It is easy to verify from the definition of $I_{i}$ that

$$
I_{i}\left(t, s ; a_{1}, \ldots, a_{i}\right)=(-1)^{i} I_{i}\left(s, t ; a_{i}, \ldots, a_{1}\right)
$$

and

$$
I_{i}\left(t, s ; a_{1}, \ldots, a_{i}\right)=\int_{s}^{t} a_{i}(u) I_{i-1}\left(t, u ; a_{1}, \ldots, a_{i-1}\right) d u .
$$

We shall need the following lemmas.

Lemma 2.1. Suppose condition (1.3) holds. If $x \in \mathcal{D}\left(\bar{L}_{n}\right)$ where $\bar{L}_{n}$ is $L_{n}$ defined by (1.2) with $\alpha=1$, is eventually of one sign, then there exist a $t_{x} \geq t_{0} \geq 0$ and an integer $\ell, 0 \leq \ell \leq n$ with $n+\ell$ even for $x(t) \bar{L}_{n} x(t)$ nonnegative eventually, or $n+\ell$ odd for $x(t) \bar{L}_{n} x(t)$ nonpositive eventually and such that for every $t \geq t_{x}$,

$$
\left\{\begin{array}{l}
\ell>0 \text { implies } x(t) \bar{L}_{k} x(t)>0, k=0,1, \ldots, \ell \\
\ell \leq n-1 \text { implies }(-1)^{\ell-k} x(t) \bar{L}_{k} x(t)>0, k=\ell, \ell+1, \ldots, n .
\end{array}\right.
$$


This lemma generalizes a well-known lemma of Kiguradze and can be proved similarly.

It will be convenient to make use of the following notation in the remainder of this paper. For any $T \geq t_{0}$ and all $t \geq T$, we let

$$
\begin{aligned}
& w_{\ell}[t, T]=a_{1}(t) \int_{T}^{t} I_{\ell-2}\left(t, s ; a_{2}, \ldots, a_{\ell-1}\right) a_{\ell}(s) I_{n-\ell-1}\left(t, s ; a_{n-1}, \ldots, a_{\ell+1}\right) d s \\
& w[t, T]=\min _{2 \leq \ell \leq n-1} w_{\ell}[t, T], \quad \text { for } 2 \leq \ell \leq n-1, \\
& \bar{w}_{\ell}[t, T]=\int_{T}^{t} I_{\ell-1}\left(t, s ; a_{1}, \ldots, a_{\ell-1}\right) a_{\ell}(s) I_{n-\ell-1}\left(t, s ; a_{n-1}, \ldots, a_{\ell+1}\right) d s, \\
& \bar{w}[t, T]=\min _{1 \leq \ell \leq n-1} \bar{w}_{\ell}[t, T] .
\end{aligned}
$$

For $t \geq s \geq T$, we let

$$
\begin{aligned}
& w_{1}[t, s]=a_{1}(s) I_{n-2}\left(t, s ; a_{n-1}, \ldots, a_{2}\right) \\
& \begin{aligned}
w_{\lambda}[t, T]=\min \left\{w_{1}[t, \lambda t], w_{\ell}[\lambda t, T], \quad 2 \leq \ell \leq n-1\right. & \text { and } 0<\lambda<1\} \\
& \text { for } t \geq T / \lambda,
\end{aligned} \\
& w_{0}[t, s]=I_{n-1}\left(t, s ; a_{n-1}, \ldots, a_{1}\right), w_{0}^{*}[t, T]=\max _{1 \leq i \leq n-1} I_{i}\left(t, T ; a_{1}, \ldots, a_{i}\right)
\end{aligned}
$$

and

$$
\bar{w}_{0}[t, s]=a_{1}(s) I_{n-2}\left(t, s ; a_{n-1}, \ldots, a_{2}\right) .
$$

The following two lemmas can be found in $[1,6]$.

Lemma 2.2. Let $x \in \mathcal{D}\left(L_{n}\right)$ be eventually positive with condition (1.3) holding. Then

$\left(i_{1}\right)$. For $1 \leq \ell \leq n-1$ and all $t \geq T \geq t_{0}$,

$$
x(t) \geq \bar{w}[t, T] L_{n-1} x(t) .
$$

$\left(i_{2}\right)$. For $2 \leq \ell \leq n-1$ and all $t \geq T \geq t_{0}$,

$$
x^{\prime}(t) \geq w[t, T] L_{n-1} x(t) .
$$

$\left(i_{3}\right)$. For $1 \leq \ell \leq n-1$, and $\lambda$ a constant with $0<\lambda<1$, there exists a $T^{*} \geq T / \lambda$ such that

$$
x^{\prime}[\lambda t] \geq w_{\lambda}[t, T] L_{n-1} x(t) \text { for } t \geq T^{*} .
$$

Lemma 2.3. Let $x \in \mathcal{D}\left(L_{n}\right)$ be eventually positive with condition (1.3) holding and let $x(t)$ satisfy (2.1) with $\ell=0$. Then for $t \geq s \geq T \geq t_{0}$,

$$
x(s) \geq w_{0}[t, s] L_{n-1} x(t)
$$

and

$$
-x^{\prime}(s) \geq \bar{w}_{0}[t, s] L_{n-1} x(t)
$$


Next, we shall need the following notation and a lemma due to Mahfoud [10]. Let

$$
\mathbb{R}_{t_{0}}= \begin{cases}\left(-\infty,-t_{0}\right] \cup\left[t_{0}, \infty\right) & \text { if } t_{0}>0 \\ (-\infty, 0) \cup(0, \infty) & \text { if } t_{0}=0\end{cases}
$$

and

$$
\begin{aligned}
C_{B}\left(\mathbb{R}_{t_{0}}\right)=\{f \in C(\mathbb{R}, \mathbb{R}): & f \text { is of bounded variation } \\
& \text { on any interval } \left.[a, b] \subset \mathbb{R}_{t_{0}}\right\} .
\end{aligned}
$$

Lemma 2.4. Suppose $t_{0}>0$ and $f \in C(\mathbb{R}, \mathbb{R})$. Then, $f \in C_{B}\left(\mathbb{R}_{t_{0}}\right)$ if and only if $f(x)=H(x) G(x)$ for all $x \in \mathbb{R}$, where $G: \mathbb{R}_{t_{0}} \rightarrow \mathbb{R}^{+}$is nondecreasing on $\left(-\infty,-t_{0}\right)$ and nonincreasing on $\left(t_{0}, \infty\right)$ and $H: \mathbb{R}_{t_{0}} \rightarrow \mathbb{R}$ is nondecreasing on $\mathbb{R}_{t_{0}}$.

We shall also need the following lemma.

Lemma $2.5([4],[11])$. Let $r(t) \in C\left([T, \infty), \mathbb{R}^{+}\right), T \geq t_{0}$. If there exists a function $w(t) \in C^{1}([T, \infty), \mathbb{R})$ such that

$$
q(t) \leq-w^{\prime}(t)-r(t) w^{2}(t) \text { for every } t \geq T,
$$

then the second order linear ordinary differential equation

$$
\left(\frac{x^{\prime}(t)}{r(t)}\right)^{\prime}+q(t) x(t)=0
$$

is nonoscillatory.

\section{Oscillation of THE EVEn ORDER EQUATION (1.1)}

Here, we shall present oscillation criteria of comparison type for equation (1.1), when $n$ is even. For this, we shall assume that there exists a function $\sigma(t) \in C^{1}\left(\left[t_{0}, \infty\right), \mathbb{R}\right)$ such that

$$
\sigma(t) \leq \inf \{t, g(t)\}, \sigma^{\prime}(t)>0 \text { for } t \geq t_{0} \text { and } \lim _{t \rightarrow \infty} \sigma(t)=\infty
$$

For all large $T \geq t_{0}$ with $\sigma(t)>T / \lambda, 0<\lambda<1$, we let

$$
w^{*}(t)=\lambda \sigma^{\prime}(t) w_{\lambda}[\sigma(t), T] \bar{w}^{\alpha-1}[\lambda \sigma(t), T] .
$$

Theorem 3.1. Let $\alpha \geq 1, f \in C\left(\mathbb{R}_{t_{0}}\right), t_{0} \geq 0$, and let $G$ and $H$ be a pair of continuous components of $f$ with $H$ being the nondecreasing one (and $G$ as described in Lemma 2.4) satisfying

$$
H(x) \operatorname{sgn} x \geq|x|^{\beta} \text { for } x \neq 0,
$$

where $\beta$ is the quotient of two positive odd integers. Moreover, assume that the conditions $(\mathrm{i})-(\mathrm{v}),(1.3)$ and (3.1) hold. Equation (1.1) is oscillatory if there exists a function $\rho(t) \in C^{1}\left(\left[t_{0}, \infty\right), \mathbb{R}^{+}\right)$and a constant $\lambda \in(0,1)$ such that for all $T \geq t_{0}$ with $\sigma(t)>T / \lambda$, the linear second order ordinary differential equation

$$
\left(\frac{1}{C(t)} z^{\prime}(t)\right)^{\prime}+p(t) z(t)=0
$$


is oscillatory, where

$$
p(t)=\rho(t) q(t) G\left(c w_{0}^{*}[g(t), T]\right)+\frac{1}{2}\left(\frac{\rho^{\prime}(t)}{\rho(t) C(t)}\right)^{\prime}-\frac{1}{4 C(t)}\left(\frac{\rho^{\prime}(t)}{\rho(t)}\right)^{2},
$$

$c$ is any positive constant, and

$$
\frac{\rho(t)}{\beta w^{*}(t)} C(t)=\left\{\begin{array}{l}
c_{1}, c_{1} \text { is any positive constant, when } \beta>\alpha, \\
1, \text { when } \beta=\alpha, \\
c_{2}\left(w_{0}^{*}[\lambda \sigma(t), T]\right)^{\beta-\alpha}, c_{2} \text { is any positive constant, when } \beta<\alpha .
\end{array}\right.
$$

Proof. Let $x(t)$ be an eventually positive solution of equation (1.1), say, $x(t)>$ 0 for $t \geq t_{0} \geq 0$. It is easy to see that the sign of $L_{n}$ and $\bar{L}_{n}$ are the same, where $\bar{L}_{n}$ is $L_{n}$ defined by (1.2) with $\alpha=1$. Since $n$ is even, there exist $t_{1} \geq t_{0}$ and an integer $\ell \in\{1,3, \ldots, n-1\}$ such that (2.1) holds. There exist a $t_{2} \geq t_{1}$ and a constant $b_{1}>0$ such that $L_{n-1} x(t) \leq b_{1}$ for $t \geq t_{2}$. Integrating the above inequality $n-1$ times, there exist a $t_{3} \geq t_{2}$ and a constant $b>0$ such that

$$
x[g(t)] \leq b w_{0}^{*}\left[g(t), t_{2}\right] \text { for } t \geq t_{3} .
$$

From equation (1.1), it follows that

$$
\begin{aligned}
-L_{n} x(t) & =q(t) f(x[g(t)])=q(t) G(x[g(t)]) H(x[g(t)]) \\
& \geq q(t) G\left(b w_{0}^{*}\left[g(t), t_{2}\right]\right) x^{\beta}[\sigma(t)] \text { for } t \geq t_{3} .
\end{aligned}
$$

Define

$$
w(t)=\rho(t) \frac{L_{n-1}^{\alpha} x(t)}{x^{\beta}[\lambda \sigma(t)]} \quad \text { for } \quad t \geq t_{3}, \quad \lambda \in(0,1) .
$$

Then, for $t \geq t_{3}$ we have

$$
\begin{aligned}
w^{\prime}(t) \leq & -\rho(t) q(t) G\left(b w_{0}^{*}\left[g(t), t_{2}\right]\right)+\frac{\rho^{\prime}(t)}{\rho(t)} w(t) \\
& -\lambda \beta \frac{\sigma^{\prime}(t)}{\rho(t)} w^{2}(t) \frac{x^{\prime}[\lambda \sigma(t)]}{L_{n-1}^{\alpha} x(t)} x^{\beta-1}[\lambda \sigma(t)] .
\end{aligned}
$$

By Lemma 2.2 there exists a $t_{4} \geq t_{3}$ such that for all $t \geq t_{4} / \lambda$,

$$
x[\lambda \sigma(t)] \geq \bar{w}\left[\lambda \sigma(t), t_{3}\right] L_{n-1} x(t)
$$

and

$$
x^{\prime}[\lambda \sigma(t)] \geq w_{\lambda}\left[\sigma(t), t_{3}\right] L_{n-1} x(t) .
$$

Using (3.7) and (3.8) in (3.6), we have

$$
\begin{aligned}
w^{\prime}(t) \leq & -\rho(t) q(t) G\left(b w_{0}^{*}\left[g(t), t_{2}\right]\right)+\frac{\rho^{\prime}(t)}{\rho(t)} w(t) \\
& -\frac{\beta}{\rho(t)} w^{*}(t) x^{\beta-\alpha}[\lambda \sigma(t)] w^{2}(t) \quad \text { for } \quad t \geq t_{4} / \lambda .
\end{aligned}
$$

Now, we consider the following three cases: 
Case 1. $\beta>\alpha$. Since $x^{\prime}(t)>0$ eventually, there exist a constant $k_{1}>0$ and a $t_{5} \geq t_{4} / \lambda$ such that

$$
x[\lambda \sigma(t)] \geq k_{1} \quad \text { for } \quad t \geq t_{5} .
$$

Thus, inequality (3.9) becomes

$$
\begin{aligned}
w^{\prime}(t) \leq & -\rho(t) q(t) G\left(b w_{0}^{*}\left[g(t), t_{2}\right]\right)+\frac{\rho^{\prime}(t)}{\rho(t)} w(t) \\
& -\frac{\beta k_{1}^{\beta-\alpha}}{\rho(t)} w^{*}(t) w^{2}(t) \quad \text { for } \quad t \geq t_{5} .
\end{aligned}
$$

Case 2. $\beta=\alpha$. In this case inequality (3.9) becomes

$$
\begin{aligned}
w^{\prime}(t) \leq & -\rho(t) q(t) G\left(b w_{0}^{*}\left[g(t), t_{2}\right]\right)+\frac{\rho^{\prime}(t)}{\rho(t)} w(t) \\
& -\frac{\beta}{\rho(t)} w^{*}(t) w^{2}(t) \quad \text { for } \quad t \geq t_{5} .
\end{aligned}
$$

Case 3. $\beta<\alpha$. As in the above proof, there exist a constant $k_{2}>0$ and a $t_{6} \geq t_{4} / \lambda$ such that

$$
x[\lambda \sigma(t)] \leq k_{2} w_{0}^{*}\left[\lambda \sigma(t), t_{4}\right] \text { for } t \geq t_{6} .
$$

Using (3.13) in (3.9), we have

$$
\begin{aligned}
w^{\prime}(t) \leq & -\rho(t) q(t) G\left(b w_{0}^{*}\left[g(t), t_{2}\right]\right)+\frac{\rho^{\prime}(t)}{\rho(t)} w(t) \\
& -\frac{\beta k_{2}^{\beta-\alpha}}{\rho(t)}\left(w_{0}^{*}\left[\lambda \sigma(t), t_{4}\right]\right)^{\beta-\alpha} w^{*}(t) w^{2}(t) \text { for } t \geq t_{6} .
\end{aligned}
$$

Choose $T_{1} \geq \max \left\{t_{5}, t_{6}\right\}$, from inequalities (3.11), (3.12) and (3.14), we get

$$
w^{\prime}(t) \leq-\rho(t) q(t) G\left(b w_{0}^{*}\left[g(t), t_{2}\right]\right)+\frac{\rho^{\prime}(t)}{\rho(t)} w(t)-C(t) w^{2}(t), \quad t \geq T_{1},
$$

where $C(t)$ here is given by

$$
\frac{\rho(t)}{\beta w^{*}(t)} C(t)=\left\{\begin{array}{l}
k_{1}^{\beta-\alpha} \text { when } \beta>\alpha, \\
1 \text { when } \beta=\alpha, \\
k_{2}^{\beta-\alpha}\left(w_{0}^{*}\left[\lambda \sigma(t), t_{4}\right]\right)^{\beta-\alpha} \text { when } \beta<\alpha .
\end{array}\right.
$$

Clearly, $C(t)>0$ for $t \geq T_{1}$. Now,

$$
\begin{aligned}
w^{\prime}(t) \leq & -\rho(t) q(t) G\left(b w_{0}^{*}\left[g(t), t_{2}\right]\right)-C(t)\left[w^{2}(t)-\frac{\rho^{\prime}(t)}{\rho(t) C(t)} w(t)\right] \\
= & -\rho(t) q(t) G\left(b w_{0}^{*}\left[g(t), t_{2}\right]\right)-C(t)\left[w(t)-\frac{\rho^{\prime}(t)}{2 \rho(t) C(t)}\right]^{2} \\
& +\frac{1}{4 C(t)}\left(\frac{\rho^{\prime}(t)}{\rho(t)}\right)^{2} \text { for } t \geq T_{1},
\end{aligned}
$$


or

$$
\begin{aligned}
\left(w(t)-\frac{\rho^{\prime}(t)}{2 \rho(t) C(t)}\right)^{\prime} \leq & -\rho(t) q(t) G\left(b w_{0}^{*}\left[g(t), t_{2}\right]\right)-\left(\frac{\rho^{\prime}(t)}{2 \rho(t) C(t)}\right)^{\prime} \\
& +\frac{1}{4 C(t)}\left(\frac{\rho^{\prime}(t)}{\rho(t)}\right)^{2}-C(t)\left[w(t)-\frac{\rho^{\prime}(t)}{2 \rho(t) C(t)}\right]^{2}, t \geq T_{1} .
\end{aligned}
$$

Set

so we have

$$
y(t)=w(t)-\frac{\rho^{\prime}(t)}{2 \rho(t) C(t)}, t \geq T_{1}
$$

$$
\begin{aligned}
y^{\prime}(t) \leq & -\left[\rho(t) q(t) G\left(b w_{0}^{*}\left[g(t), t_{2}\right]\right)+\left(\frac{\rho^{\prime}(t)}{2 \rho(t) C(t)}\right)^{\prime}\right. \\
& \left.-\frac{1}{4 C(t)}\left(\frac{\rho^{\prime}(t)}{\rho(t)}\right)^{2}\right]-C(t) y^{2}(t) \text { for } t \geq T_{1},
\end{aligned}
$$

or

$$
y^{\prime}(t) \leq-p(t)-C(t) y^{2}(t) \text { for } t \geq T_{1},
$$

where $p(t)$ is given by

$$
p(t)=\rho(t) q(t) G\left(b w_{0}^{*}\left[g(t), t_{2}\right]\right)+\left(\frac{\rho^{\prime}(t)}{2 \rho(t) C(t)}\right)^{\prime}-\frac{1}{4 C(t)}\left(\frac{\rho^{\prime}(t)}{\rho(t)}\right)^{2} .
$$

Applying Lemma 2.5 to inequality (3.16), we conclude that the linear equation (3.3) is nonoscillatory, which is a contradiction. This completes the proof.

Next, we shall consider equation (1.1) with $0<\alpha \leq 1$ and prove the following result.

Theorem 3.2. Let $0<\alpha \leq 1, f \in C\left(\mathbb{R}_{t_{0}}\right), t_{0} \geq 0$ and let $G$ and $H$ be a pair of continuous components of $f$ with $H$ being the nondecreasing one (and $G$ as described in Lemma 2.4). Moreover, assume that the conditions (i)-(v), (1.3), (3.1) and (3.2) hold. Equation (1.1) is oscillatory if there exist a function $\rho(t) \in C^{1}\left(\left[t_{0}, \infty\right), \mathbb{R}^{+}\right)$and a constant $\lambda, 0<\lambda<1$ such that for all $T \geq t_{0}$ with $\sigma(t)>T / \lambda$, the linear second order ordinary differential equation

$$
\left(\frac{1}{Q^{*}(t)} y^{\prime}(t)\right)^{\prime}+P(t) y(t)=0
$$

is oscillatory, where

$$
\begin{aligned}
& Q^{*}(t)=\lambda \beta \sigma^{\prime}(t) \rho^{-1 / \alpha}(t) w_{\lambda}[\sigma(t), T] Q^{(1 / \alpha)-1}(t) c(t) \\
& Q(t)=\rho(t) \int_{t}^{\infty} q(s) G\left(c^{*} w_{0}^{*}[g(s), T]\right) d s \\
& P(t)=\rho(t) q(t) G\left(c^{*} w_{0}^{*}[g(t), T]\right)+\frac{1}{2}\left(\frac{\rho^{\prime}(t)}{\rho(t) Q^{*}(t)}\right)^{\prime}-\frac{1}{4 Q^{*}(t)}\left(\frac{\rho^{\prime}(t)}{\rho(t)}\right)^{2}
\end{aligned}
$$


$c^{*}$ is any positive constant and

$$
c(t)=\left\{\begin{array}{l}
c_{1}, c_{1}>0 \text { is any constant, when } \beta>\alpha, \\
1, \text { when } \beta=\alpha, \\
c_{2}\left(w_{0}^{*}[\lambda \sigma(t), T]\right)^{(\beta / \alpha)-1}, c_{2}>0 \text { is any constant, when } \beta<\alpha .
\end{array}\right.
$$

Proof. Let $x(t)$ be an eventually positive solution of equation (1.1), say, $x(t)>$ 0 for $t \geq t_{0} \geq 0$. Define the function $w(t)$ as in the proof of Theorem 3.1 and obtain $(3.4)-(3.8)$ for $t \geq t_{4}$. Now, one can easily obtain

$$
\begin{aligned}
w^{\prime}(t) \leq & -\rho(t) q(t) G\left(b w_{0}^{*}\left[g(t), t_{2}\right]\right)+\frac{\rho^{\prime}(t)}{\rho(t)} w(t) \\
& -\lambda \beta \sigma^{\prime}(t) \rho^{-1 / \alpha}(t) w_{\lambda}\left[\sigma(t), t_{3}\right] w^{2}(t) w^{(1 / \alpha)-1}(t) x^{(\beta / \alpha)-1}[\lambda \sigma(t)] .
\end{aligned}
$$

As a result

$$
\left(\frac{w(t)}{\rho(t)}\right)^{\prime}=\frac{1}{\rho(t)}\left[w^{\prime}(t)-\frac{\rho^{\prime}(t)}{\rho(t)} w(t)\right] \leq-q(t) G\left(b w_{0}^{*}\left[g(t), t_{2}\right]\right), t \geq t_{3} .
$$

Integrating (3.19) from $t \geq t_{3}$ to $u$ and letting $u \rightarrow \infty$, we have

$$
w(t) \geq Q(t) \text { for } t \geq t_{3} \text {. }
$$

Using (3.20) in (3.18), we obtain

$$
\begin{aligned}
w^{\prime}(t) \leq & -\rho(t) q(t) G\left(b w_{0}^{*}\left[g(t), t_{2}\right]\right)+\frac{\rho^{\prime}(t)}{\rho(t)} w(t) \\
& -\frac{Q^{*}(t)}{c(t)} x^{(\beta / \alpha)-1}[\lambda \sigma(t)] w^{2}(t) \quad \text { for } \quad t \geq t_{4} .
\end{aligned}
$$

The rest of the proof is similar to that of Theorem 3.1 and hence omitted.

The following corollaries are immediate.

Corollary 3.1. Let $\alpha \geq 1, f \in C\left(\mathbb{R}_{t_{0}}\right), t_{0} \geq 0$, and let $G$ and $H$ be a pair of continuous components of $f$ with $H$ being the nondecreasing one (and $G$ as described in Lemma 2.4). Moreover, assume that the conditions (i)-(v), (1.3), (3.1) and (3.2) hold. If for some constant $\lambda, 0<\lambda<1$, and all large $T \geq t_{0}$ with $\sigma(t)>T / \lambda$, the second order linear equation

$$
\left(\frac{1}{a(t)} y^{\prime}(t)\right)^{\prime}+p(t) y(t)=0
$$

is oscillatory, where

$$
\begin{aligned}
& p(t)=q(t) G\left(c w_{0}^{*}[g(t), T]\right), \quad c \text { is any positive constant, } \\
& a(t)=\beta w^{*}(t) c(t)
\end{aligned}
$$

and

$$
c(t)=\left\{\begin{array}{l}
c_{1}, c_{1}>0 \text { is any constant, when } \beta>\alpha, \\
1, \text { when } \beta=\alpha, \\
c_{2}\left(w_{0}^{*}[\lambda \sigma(t), T]\right)^{\beta-\alpha}, c_{2}>0 \text { is any constant, when } \beta<\alpha,
\end{array}\right.
$$


then equation (1.1) is oscillatory.

Corollary 3.2. Let $0<\alpha \leq 1, f \in C\left(\mathbb{R}_{t_{0}}\right), t_{0} \geq 0$, and let $G$ and $H$ be $a$ pair of continuous components of $f$ with $H$ being the nondecreasing one (and $G$ as described in Lemma 2.4). In addition, assume that the conditions (i)-(v), (1.3), (3.1) and (3.2) hold. Equation (1.1) is oscillatory if for some constant $\lambda, 0<\lambda<1$, and all large $T \geq t_{0}$ with $\sigma(t)>T / \lambda$, the second order linear equation

$$
\left(\frac{1}{\bar{a}(t)} z^{\prime}(t)\right)^{\prime}+p(t) z(t)=0
$$

is oscillatory, where $p(t)$ is as in equation (3.22),

$$
\bar{a}(t)=\lambda \beta \sigma^{\prime}(t) w_{\lambda}[\sigma(t), T]\left(\int_{t}^{\infty} p(s) d s\right)^{(1 / \alpha)-1} \bar{c}(t)
$$

and

$$
\bar{c}(t)=\left\{\begin{array}{l}
c_{1}, c_{1}>0 \text { is any constant, when } \beta>\alpha, \\
1, \text { when } \beta=\alpha, \\
c_{2}\left(w_{0}^{*}[\lambda \sigma(t), T]\right)^{(\beta / \alpha)-1}, c_{2}>0 \text { is any constant, when } \beta<\alpha .
\end{array}\right.
$$

The proof of Corollaries 3.1 and 3.2 can be obtained from that of Theorems 3.1 and 3.2, respectively, by letting $\rho(t)=1$. The details are omitted.

Corollary 3.3. Let $\alpha=1, f \in C\left(\mathbb{R}_{t_{0}}\right), t_{0} \geq 0$, and let $G$ and $H$ be a pair of continuous components of $f$ with $H$ being the nondecreasing one (and $G$ as described in Lemma 2.4). Moreover, assume that the conditions (i)-(v), (1.3), (3.1) and (3.2) hold. Equation (1.1) is oscillatory if there exist a function $\rho(t) \in C^{1}\left(\left[t_{0}, \infty\right), \mathbb{R}^{+}\right)$and a constant $\lambda, 0<\lambda<1$, such that for all large $T \geq t_{0}$ with $\sigma(t)>T / \lambda$, the second order linear equation

$$
\left(\frac{1}{r(t)} y^{\prime}(t)\right)^{\prime}+\bar{p}(t) y(t)=0
$$

is oscillatory, where

$$
\begin{aligned}
& r(t)=\lambda \beta \frac{\sigma^{\prime}(t)}{\rho(t)} w_{\lambda}[\sigma(t), T] c(t), \\
& \bar{p}(t)=\rho(t) q(t) G\left(c^{*} w_{0}^{*}[g(t), T]\right)+\frac{1}{2}\left(\frac{\rho^{\prime}(t)}{r(t) \rho(t)}\right)^{\prime}-\frac{1}{4 r(t)}\left(\frac{\rho^{\prime}(t)}{\rho(t)}\right)^{2},
\end{aligned}
$$

$c^{*}>0$ is any constant, and

$$
c(t)=\left\{\begin{array}{l}
c_{1}, c_{1}>0 \text { is any constant, when } \beta>1, \\
1, \text { when } \beta=1, \\
c_{2}\left(w_{0}^{*}[\lambda \sigma(t), T]\right)^{\beta-1}, c_{2}>0 \text { is any constant, when } \beta<1 .
\end{array}\right.
$$


Corollary 3.4. Let $\alpha=1, f \in C\left(\mathbb{R}_{t_{0}}\right), t_{0} \geq 0$, and let $G$ and $H$ be a pair of continuous components of $f$ with $H$ being the nondecreasing one (and $G$ as described in Lemma 2.4). In addition, suppose that the conditions (i)-(v), (1.3), (3.1) and (3.2) hold. Equation (1.1) is oscillatory if for some constant $\lambda$, $0<\lambda<1$, and all large $T \geq t_{0}$ with $\sigma(t)>T / \lambda$, the second order linear equation

$$
\left(\frac{1}{\bar{r}(t)} y^{\prime}(t)\right)^{\prime}+p(t) y(t)=0
$$

is oscillatory, where $p(t)$ is as in equation (3.22),

$$
\bar{r}(t)=\lambda \beta \sigma^{\prime}(t) w_{\lambda}[\sigma(t), T] c(t)
$$

and $c(t)$ is as in Corollary 3.3.

Remark 1 . We note that the above results can be applied to equations of type (1.1) with $f$ being any of the following functions:

(i) $f(x)$ is a nondecreasing function. In this case we let $f(x)=H(x)$ and $G(x)=1$,

(ii) $f(x)=\frac{|x|^{\beta-1} x}{1+|x|^{\gamma}}$, where $\beta$ and $\gamma$ are positive constants,

(iii) $f(x)=|x|^{\beta-1} x \exp \left(-|x|^{\gamma}\right)$, where $\beta$ and $\gamma$ are positive constants,

(iv) $f(x)=|x|^{\beta-1} x \operatorname{sech} x$, where $\beta$ is a positive constant.

Remark 2. In all of the above results we require the oscillation of linear second order ordinary differential equations of the form

$$
\left(a(t) x^{\prime}(t)\right)^{\prime}+q(t) x(t)=0,
$$

where $a(t) \in C\left(\left[t_{0}, \infty\right), \mathbb{R}^{+}\right)$and $q(t) \in C\left(\left[t_{0}, \infty\right), \mathbb{R}\right)$, so, it would be of interest if we give some oscillation criteria for equation (3.26).

Each of the following conditions is sufficient for the oscillation of (3.26).

$\left(\mathrm{I}_{1}\right) \int^{\infty} \frac{1}{a(s)} d s=\infty$ and $\int^{\infty} q(s) d s=\infty$,

$\left(\mathrm{I}_{2}\right) \liminf _{t \rightarrow \infty} A(t) Q(t)>\frac{1}{4}$, where $A(t)=\int_{t_{0}}^{t} \frac{1}{a(s)} d s$ and $Q(t)=\int_{t}^{\infty} q(s) d s$,

$\left(\mathrm{I}_{3}\right)$ there exists a function $\rho(t) \in C^{1}\left(\left[t_{0}, \infty\right), \mathbb{R}^{+}\right)$such that

$$
\limsup _{t \rightarrow \infty} \int_{t_{0}}^{t}\left[\rho(s) q(s)-\frac{\left(\rho^{\prime}(s)\right)^{2}}{4 a(s) \rho(s)}\right] d s=\infty
$$

For more oscillation results for equation (3.26), we refer the reader to the monograph by Agarwal et. al. [4]. 


\section{Oscillation of the Odd Order Equation (1.1)}

In this section, we shall present oscillation criteria of comparison type for equation (1.1) when $n$ is odd.

Theorem 4.1. Let $\alpha \geq 1, f \in C\left(\mathbb{R}_{t_{0}}\right), t_{0} \geq 0$, and let $G$ and $H$ be a pair of continuous components of $f$ with $H$ being the nondecreasing one (and $G$ as described in Lemma 2.4). Moreover, suppose that the conditions (i)-(v), (1.3), (3.1) and (3.2) hold. Every unbounded solution of equation (1.1) is oscillatory if there exists a function $\rho(t) \in C^{1}\left(\left[t_{0}, \infty\right), \mathbb{R}^{+}\right)$such that for all large $T \geq t_{0}$ with $\sigma(t)>T$ and all constant $c>0$, the linear second order ordinary differential equation

$$
\left(\frac{1}{r(t)} y^{\prime}(t)\right)^{\prime}+p(t) y(t)=0
$$

is oscillatory, where

$$
\begin{gathered}
r(t)=\beta \frac{\sigma^{\prime}(t)}{\rho(t)} w[\sigma(t), T] \bar{w}^{\alpha-1}[\sigma(t), T] c(t) \\
p(t)=\rho(t) q(t) G\left(c w_{0}^{*}[g(t), T]\right)+\frac{1}{2}\left(\frac{\rho^{\prime}(t)}{r(t) \rho(t)}\right)^{\prime}-\frac{1}{4 r(t)}\left(\frac{\rho^{\prime}(t)}{\rho(t)}\right)^{2}
\end{gathered}
$$

and

$$
c(t)=\left\{\begin{array}{l}
c_{1}, c_{1}>0 \text { is any constant, when } \beta>\alpha, \\
1, \text { when } \beta=\alpha, \\
c_{2}\left(w_{0}^{*}[\sigma(t), T]\right)^{\beta-\alpha}, c_{2}>0 \text { is any constant, when } \beta<\alpha .
\end{array}\right.
$$

Proof. Let $x(t)$ be an unbounded eventually positive solution of equation (1.1), say, $x(t)>0$ for $t \geq t_{0} \geq 0$. By Lemma 2.1 there exist an integer $\ell \in\{2,4, \ldots, n-1\}$ and a $t_{1} \geq t_{0}$ such that (2.1) holds. As in the proof of Theorem 3.1, we obtain (3.5) for $t \geq t_{3}$. Define

$$
w(t)=\rho(t) \frac{L_{n-1}^{\alpha} x(t)}{x^{\beta}[\sigma(t)]}, t \geq t_{3} .
$$

Then, for $t \geq t_{3}$ we have

$$
\begin{aligned}
w^{\prime}(t) \leq & -\rho(t) q(t) G\left(b w_{0}^{*}\left[g(t), t_{2}\right]\right)+\frac{\rho^{\prime}(t)}{\rho(t)} w(t) \\
& -\beta \frac{\sigma^{\prime}(t)}{\rho(t)} \frac{x^{\prime}[\sigma(t)]}{L_{n-1}^{\alpha} x(t)} x^{\beta-1}[\sigma(t)] w^{2}(t) .
\end{aligned}
$$

By Lemma 2.2, there exists a $t_{4} \geq t_{3}$ such that $\sigma(t)>t_{3}$ for $t \geq t_{4}$,

$$
x[\sigma(t)] \geq \bar{w}\left[\sigma(t), t_{3}\right] L_{n-1} x(t), \quad t \geq t_{4},
$$

and

$$
x^{\prime}[\sigma(t)] \geq w\left[\sigma(t), t_{3}\right] L_{n-1} x(t), \quad t \geq t_{4} .
$$


Using (4.3) and (4.4) in (4.2) for $t \geq t_{4}$, we have

$$
\begin{aligned}
w^{\prime}(t) \leq & -\rho(t) q(t) G\left(b w_{0}^{*}\left[g(t), t_{2}\right]\right)+\frac{\rho^{\prime}(t)}{\rho(t)} w(t) \\
& -\beta \frac{\sigma^{\prime}(t)}{\rho(t)} w\left[\sigma(t), t_{3}\right] \bar{w}^{\alpha-1}\left[\sigma(t), t_{3}\right] x^{\beta-\alpha}[\sigma(t)] w^{2}(t) .
\end{aligned}
$$

The rest of the proof is similar to that of Theorem 3.1 and hence omitted.

Theorem 4.2. Let $0<\alpha \leq 1, f \in C\left(\mathbb{R}_{t_{0}}\right), t_{0} \geq 0$, and let $G$ and $H$ be a pair of continuous components of $f$ with $H$ being the nondecreasing one (and $G$ as described in Lemma 2.4). Moreover, assume that the conditions (i)-(v), (1.3), (3.1) and (3.2) hold. Every unbounded solution of equation (1.1) is oscillatory if there exists a function $\rho(t) \in C^{1}\left(\left[t_{0}, \infty\right), \mathbb{R}^{+}\right)$such that for all large $T \geq t_{0}$ with $\sigma(t)>T$, the linear second order ordinary differential equation

$$
\left(\frac{1}{\bar{r}(t)} y^{\prime}(t)\right)^{\prime}+\bar{p}(t) y(t)=0
$$

is oscillatory, where

$$
\begin{gathered}
\bar{r}(t)=\beta \sigma^{\prime}(t) \rho^{-1 / \alpha}(t) w[\sigma(t), T] Q^{(1 / \alpha)-1}(t) c(t) \\
Q(t)=\rho(t) \int_{t}^{\infty} q(s) G\left(c w_{0}^{*}[g(s), T]\right) d s, \quad c \text { is any positive constant, } \\
\bar{p}(t)=\rho(t) q(t) G\left(c w_{0}^{*}[g(t), T]\right)+\frac{1}{2}\left(\frac{\rho^{\prime}(t)}{\bar{r}(t) \rho(t)}\right)^{\prime}-\frac{1}{4 \bar{r}(t)}\left(\frac{\rho^{\prime}(t)}{\rho(t)}\right)^{2}
\end{gathered}
$$

and

$$
c(t)=\left\{\begin{array}{l}
c_{1}, c_{1}>0 \text { is any constant, when } \beta>\alpha, \\
1, \text { when } \beta=\alpha, \\
c_{2}\left(w_{0}^{*}[\sigma(t), T]\right)^{(\beta / \alpha)-1}, c_{2}>0 \text { is any constant, when } \beta<\alpha .
\end{array}\right.
$$

The proof can be modelled on that of Theorem 3.2 and hence omitted.

Next, we are concerned with the oscillatory behavior of all bounded solutions of equation (1.1).

For any function $g(t) \in C\left(\left[t_{0}, \infty\right), \mathbb{R}\right)$, we put

$$
\mathcal{R}_{g}=\left\{t \in\left[t_{0}, \infty\right), g(t)<t\right\} .
$$

We also introduce the notation: $\tau(t)=\max \left\{\min \{s, g(s)\}: t_{0} \leq s \leq t\right\}$ and note that the following inequality holds $g(s) \leq \tau(t)$ for $\tau(t)<s<t$.

Now, we present the following results.

Theorem 4.3. Let $f \in C\left(\mathbb{R}_{t_{0}}\right), t_{0} \geq 0$, and let $G$ and $H$ be a pair of continuous components of $f$ with $H$ being the nondecreasing one (and $G$ as described in Lemma 2.4). In addition, suppose that the conditions (i)-(v), (1.3), 
(3.1) and (3.2) hold. Every bounded solution of equation (1.1) is oscillatory if for every constant $\xi>0$ either

$$
\limsup _{t \rightarrow \infty} \int_{\tau(t)}^{t} q(s) w_{0}^{\alpha}[\tau(t), g(s)] d s>\xi \quad \text { when } \alpha=\beta
$$

or

$$
\limsup _{t \rightarrow \infty} \int_{\tau(t)}^{t} q(s) w_{0}^{\beta}[\tau(t), g(s)] d s>0 \quad \text { when } \alpha>\beta .
$$

Remark 3. Of course in Theorem 4.3 for (4.6) to hold we need the left hand side of the inequality in (4.6) to be infinity. However we can improve (4.6) if we know a little more about $G$. For example if $G$ is a constant (say $G(x)=a_{0}>0$ ) then it is enough to assume (4.6) only for $\xi=\frac{1}{a_{0}}$.

Proof. Let $x(t)$ be a bounded nonoscillatory solution of equation (1.1), say, $x(t)>0$ for $t \geq t_{0} \geq 0$. By Lemma 2.1, there exists a $t_{1} \geq t_{0}$ such that $\inf \left\{g(t): t \geq t_{1}\right\}>t_{0}$ and

$$
(-1)^{i} L_{j} x(t)>0,0 \leq j \leq n \text { on }\left[t_{1}, \infty\right) .
$$

By Lemma 2.3 there exists a $t_{2} \geq t_{1}$ such that

$$
x[g(s)] \geq w_{0}[\tau(t), g(s)] L_{n-1} x[\tau(t)] \text { for } t \geq s \geq t_{2} .
$$

Since $x(t)$ is bounded on $\left[t_{0}, \infty\right)$, there exist a constant $b>0$ and a $t_{3} \geq t_{2}$ such that

$$
x[g(t)] \leq b \text { for } t \geq t_{3} .
$$

Using condition (3.2), (4.9) and (4.10) in equation (1.1), we have

$$
\begin{aligned}
-\frac{d}{d s}\left(L_{n-1} x(s)\right)^{\alpha} & =q(s) f(x[g(s)])=q(s) G(x[g(s)]) H(x[g(s)]) \\
& \geq q(s) G(b) x^{\beta}[g(s)] \geq q(s) G(b) w_{0}^{\beta}[\tau(t), g(s)] L_{n-1}^{\beta} x[\tau(t)] \\
& \text { for } t \geq s \geq t_{3} .
\end{aligned}
$$

Integrating both sides of the above inequality from $\tau(t)$ to $t$, we have

$$
L_{n-1}^{\alpha} x[\tau(t)] \geq L_{n-1}^{\beta} x[\tau(t)] \int_{\tau(t)}^{t} q(s) w_{0}^{\beta}[\tau(t), g(s)] G(b) d s .
$$

Now, we consider the following two cases:

Case 1. $\alpha=\beta$. In this case (4.11) reduces to

$$
L_{n-1}^{\alpha} x[\tau(t)]\left[\int_{\tau(t)}^{t} q(s) w_{0}^{\alpha}[\tau(t), g(s)] G(b) d s-1\right] \leq 0 \quad \text { for } \quad t \geq t_{1} .
$$

But this is inconsistent with (4.6). 
Case 2. $\alpha>\beta$. It follows from (4.11) that

$$
L_{n-1}^{\alpha-\beta} x[\tau(t)] \geq \int_{\tau(t)}^{t} q(s) w_{0}^{\beta}[\tau(t), g(s)] G(b) d s .
$$

Taking lim sup of both sides of (4.12) as $t \rightarrow \infty$, we see that the left-hand side approaches zero, which contradicts (4.7). This completes the proof.

Theorem 4.4. Let the hypotheses of Theorem 4.3 hold with $g(t)<t$ for $t \geq t_{0}$ and conditions (4.6) and (4.7) are replaced, respectively, by

$$
\begin{gathered}
\limsup _{t \rightarrow \infty} \int_{g(t)}^{t} I_{n-2}\left(v, g(t) ; a_{n-2}, \ldots, a_{1}\right) a_{n-1}(v)\left(\int_{v}^{t} q(u) d u\right)^{1 / \alpha} d v>\xi, \\
\text { when } \alpha=\beta
\end{gathered}
$$

$\xi>0$ is any constant, or

$$
\begin{gathered}
\limsup _{t \rightarrow \infty} \int_{g(t)}^{t} I_{n-2}\left(v, g(t) ; a_{n-2}, \ldots, a_{1}\right) a_{n-1}(v)\left(\int_{v}^{t} q(u) d u\right)^{1 / \alpha} d v>0 \\
\text { when } \alpha>\beta .
\end{gathered}
$$

Then the conclusion of Theorem 4.3 holds.

Proof. Let $x(t)$ be a bounded nonoscillatory solution of equation (1.1), say, $x(t)>0$ for $t \geq t_{0} \geq 0$. There exists a $t_{1} \geq t_{0}$ such that (4.8) holds for $t \geq t_{1}$. Choose a $t_{2}>t_{1}$ such that $\inf \left\{g(t): t \geq t_{2}\right\}>t_{1}$. By Taylor's formula with remainder it is easy to see that

$$
\begin{gathered}
x[g(t)] \geq \int_{g(t)}^{t} I_{n-2}\left(v, g(t) ; a_{n-2}, \ldots, a_{1}\right) a_{n-1}(v)\left(L_{n-1} x(v)\right) d v \\
\text { for } t>g(t) \geq t_{2} .
\end{gathered}
$$

As in the proof of Theorem 4.3, we obtain (4.10) and from equation (1.1), we have

$$
-\frac{d}{d t}\left(L_{n-1}^{\alpha} x(t)\right) \geq q(t) G(b) x^{\beta}[g(t)] \text { for } t \geq t_{3} \geq t_{2}
$$

Integrating (4.16) from $v$ to $t$, we obtain

$$
L_{n-1} x(v) \geq\left(\int_{v}^{t} q(u) G(b) x^{\beta}[g(u)] d u\right)^{1 / \alpha} \text { for } t \geq v \geq t_{3} .
$$

Substituting (4.17) in (4.15), we have

$$
x[g(t)] \geq G(b) \int_{g(t)}^{t} I_{n-2}\left(v, g(t) ; a_{n-1}, \ldots, a_{1}\right) a_{n-1}(v)\left(\int_{v}^{t} q(u) d u\right)^{1 / \alpha} x^{\beta / \alpha}[g(t)] d v .
$$


The rest of the proof is similar to that of Theorem 4.3 and hence omitted.

Theorem 4.5. Let the hypotheses of Theorem 4.3 hold with $0<\beta<\alpha$ and condition (4.7) is replaced by

$$
\int_{\mathcal{R}_{g}} q(s) w_{0}^{\beta}[s, g(s)] d s=\infty .
$$

Then the conclusion of Theorem 4.3 holds.

Proof. Let $x(t)$ be a bounded nonoscillatory solution of equation (1.1), say, $x(t)>0$ for $t \geq t_{0} \geq 0$. There exists a $t_{1} \geq t_{0}$ such that (4.8) holds for $t \geq t_{1}$. Choose a $t_{2} \geq t_{1}$ such that $\inf \left\{g(t): t \geq t_{2}\right\}>t_{1}$. As in the proof of Theorem 4.3, we obtain (4.10) and

$$
x[g(t)] \geq w_{0}[t, g(t)] L_{n-1} x(t) \quad \text { for } \quad t \in \mathcal{R}_{g} \cap\left[t_{2}, \infty\right) .
$$

Using condition (3.2), (4.10) and (4.19) and letting $u(t)=L_{n-1} x(t)>0$ on $\mathcal{R}_{g} \cap\left[t_{2}, \infty\right)$, we have

$$
-\frac{d u(t)}{d t} \geq q(t) G(b) w_{0}^{\beta}[t, g(t)] u^{\beta / \alpha}(t) \quad \text { for } \quad t \in \mathcal{R}_{g} \cap\left[t_{2}, \infty\right) .
$$

Choose $T \geq t_{2}$ arbitrarily. Dividing both sides of the above inequality by $u^{\beta / \alpha}(t)$ and integrating on $\mathcal{R}_{g} \cap\left[t_{2}, T\right]$, we find

$$
\begin{aligned}
\int_{\mathcal{R}_{g} \cap\left[t_{2}, T\right]} q(s) w_{0}^{\beta}[s, g(s)] d s & \leq \frac{1}{G(b)} \int_{T}^{t_{2}} u^{-\beta / \alpha}(s) u^{\prime}(s) d s=\frac{1}{G(b)} \int_{u(T)}^{u\left(t_{2}\right)} u^{-\beta / \alpha} d u \\
& =\frac{1}{G(b)}\left(\frac{\alpha}{\alpha-\beta}\right)\left[u^{1-(\beta / \alpha)}\left(t_{2}\right)-u^{1-(\beta / \alpha)}(T)\right] .
\end{aligned}
$$

Letting $T \rightarrow \infty$, we conclude that

$$
\int_{\mathcal{R}_{g} \cap\left[t_{2}, \infty\right)} q(s) w_{0}^{\beta}[s, g(s)] d s \leq \frac{1}{G(b)}\left(\frac{\alpha}{\alpha-\beta}\right) u^{1-(\beta / \alpha)}\left(t_{2}\right)<\infty,
$$

which contradicts condition (4.18). This completes the proof.

Now, we are ready to state oscillation theorems for the odd order equation (1.1).

Theorem 4.6. Let $\alpha \geq 1, f \in C\left(\mathbb{R}_{t_{0}}\right), t_{0} \geq 0$, and let $G$ and $H$ be a pair of continuous components of $f$ with $H$ being the nondecreasing one (and $G$ as described in Lemma 2.4). Moreover, assume that conditions (i)-(v), (1.3), (3.1) and (3.2) hold with $0<\beta \leq \alpha$. Equation (1.1) is oscillatory if there exists a function $\rho(t) \in C^{1}\left(\left[t_{0}, \infty\right), \mathbb{R}^{+}\right)$such that for all large $T \geq t_{0}$ with $\sigma(t)>T$ and all positive constants $c$ and $\xi$, the linear equation (4.1) is oscillatory where $r(t)$ and $p(t)$ are as in Theorem 4.1 and

$$
c(t)=\left\{\begin{array}{l}
1 \text { when } \alpha=\beta, \\
c_{2}\left(w_{0}^{*}[\sigma(t), T]\right)^{\beta-\alpha}, \text { where } c_{2}>0 \text { is any constant, when } \alpha>\beta
\end{array}\right.
$$


and condition (4.6) or (4.13) holds when $\alpha=\beta$ or condition (4.7) or (4.14) or (4.18) holds when $\alpha>\beta$.

Theorem 4.7. Let $\alpha \leq 1, f \in C\left(\mathbb{R}_{t_{0}}\right), t_{0} \geq 0$, and let $G$ and $H$ be a pair of continuous components of $f$ with $H$ being the nondecreasing one (and $G$ as described in Lemma 2.4). In addition, assume that conditions (i)-(v), (1.3), (3.1) and (3.2) hold with $0<\beta \leq \alpha$. Equation (1.1) is oscillatory if there exists a function $\rho(t) \in C^{1}\left(\left[t_{0}, \infty\right), \mathbb{R}^{+}\right)$such that for all large $T \geq t_{0}$ with $\sigma(t)>T$ and all positive constants $c$ and $\xi$, the linear equation (4.5) is oscillatory, where $\bar{r}(t)$ and $\bar{p}(t)$ are as in Theorem 4.2 and

$$
c(t)=\left\{\begin{array}{l}
1 \text { when } \alpha=\beta, \\
c_{2}\left(w_{0}^{*}[\sigma(t), T]\right)^{(\beta / \alpha)-1}, \text { where } c_{2}>0 \text { is any constant, when } \alpha>\beta
\end{array}\right.
$$

and either condition (4.6) or (4.13) holds when $\alpha=\beta$ or condition (4.7) or (4.14) or (4.18) holds when $\alpha>\beta$.

Next, we state an interesting special case of Theorem 4.6.

Corollary 4.1. Let $\alpha=1$, conditions (i)-(v), (1.3) and (3.1) hold and $f$ satisfies the condition

$$
f(x) \operatorname{sgn} x \geq|x|^{\beta} \text { for } x \neq 0,
$$

where $\beta$ is the quotient of two positive odd integers. If for all large $T \geq t_{0}$ with $\sigma(t)>T$, the linear equation

$$
\left(\frac{1}{a(t)} y^{\prime}(t)\right)^{\prime}+p(t) y(t)=0
$$

is oscillatory, where

$$
a(t)=\beta \sigma^{\prime}(t) w[\sigma(t), T] c(t), p(t)=q(t)
$$

and

$$
c(t)=\left\{\begin{array}{l}
1 \text { when } \beta=1, \\
c_{2}\left(w_{0}^{*}[\sigma(t), T]\right)^{\beta-1}, \text { where } c_{2}>0 \text { is any constant, when } \beta<1 .
\end{array}\right.
$$

Moreover, assume that either

$$
\limsup _{t \rightarrow \infty} \int_{\tau(t)}^{t} q(s) w_{0}^{\alpha}[\tau(t), g(s)] d s>1
$$

or $g(t)<t$ for $t \geq t_{0}$, and

$$
\limsup _{t \rightarrow \infty} \int_{g(t)}^{t} I_{n-2}\left(v, g(t) ; a_{n-2}, \ldots, a_{1}\right) a_{n-1}(v)\left(\int_{v}^{t} q(s) d s\right)^{1 / \alpha} d s>1 \text { when } \beta=1
$$

or condition (4.7) or (4.14) or (4.18) holds when $0<\beta<1$, then equation (1.1) is oscillatory. 


\section{Applications}

In this section, we shall extend our previous results to neutral equations of the type

$$
L_{n}(x(t)+p(t) x[h(t)])+q(t) f(x[g(t)])=0,
$$

where $q(t), g(t), f(x)$ and $L_{n}$ are as in equation (1.1), $p(t) \in C\left(\left[t_{0}, \infty\right), \mathbb{R}^{+} \cup\right.$ $\{0\})$ and $h(t) \in C\left(\left[t_{0}, \infty\right), \mathbb{R}\right), h^{\prime}(t)>0$ for $t \geq t_{0}$ and $\lim _{t \rightarrow \infty} h(t)=\infty$.

Set

$$
y(t)=x(t)+p(t) x[h(t)], t \geq t_{0} \geq 0 .
$$

Then for $t \geq t_{0}$, we have

$$
\frac{d}{d t}\left(L_{n-1}^{\alpha} y(t)\right)+q(t) f(x[g(t)])=0 .
$$

Now, if $x(t)$ is a nonoscillatory solution of equation (5.1), say, $x(t)>0$, $x[h(t)]>0$ and $x[g(t)]>0$ for $t \geq t_{0} \geq 0$. Then $y(t)>0$ for $t \geq t_{0}$ and there exists a $t_{1} \geq t_{0}$ such that either $\left(\mathrm{I}_{1}\right) \cdot y^{\prime}(t)>0$, or $\left(\mathrm{I}_{2}\right) \cdot y^{\prime}(t)<0$ for $t \geq t_{1}$.

$\left(\mathrm{I}_{1}\right)$. Suppose $y^{\prime}(t)>0$ for $t \geq t_{1}$. This case occurs when $n$ is even, or when we are concerned with unbounded solutions of odd order equation (5.1).

We shall examine the following two cases for $p(t)$ and $h(t)$ :

$$
(I) .\{0 \leq p(t) \leq 1, h(t)<t\} \text { and }(I I) .\{p(t) \geq 1, h(t)>t\} .
$$

For the case (I), we assume that

$$
0 \leq p(t) \leq 1, h(t)<t \text { and } p(t) \not \equiv 1 \text { eventually. }
$$

Now, we have for $t \geq t_{1}$,

$$
\begin{aligned}
x(t) & =y(t)-p(t) x[h(t)]=y(t)-p(t)[y[h(t)]-p[h(t)] x[h \circ h(t)]] \\
& \geq y(t)-p(t) y[h(t)] \geq(1-p(t)) y(t) .
\end{aligned}
$$

Next, if $f \in C\left(\mathbb{R}_{t_{0}}\right), t_{0} \geq 0$, then $f(x)=G(x) H(x)$. Now, if condition (3.2) holds, then equation (5.3) becomes

$$
\begin{aligned}
0 & =L_{n} y(t)+q(t) f(x[g(t)]) \\
& =L_{n} y(t)+q(t) G(x[g(t)]) H(x[g(t)]) \\
& \geq L_{n} y(t)+q(t) G(x[g(t)])(1-p[g(t)])^{\beta} y^{\beta}[g(t)] \text { for } t \geq t_{1} .
\end{aligned}
$$

Using the fact that $x(t) \geq y(t)$, there exist a constant $b>0$ and a $t_{2} \geq t_{1}$ such that

$$
y[g(t)] \leq b w_{0}^{*}\left[g(t), t_{2}\right] \text { for } t \geq t_{3} \text { for some } t_{3} \geq t_{2} .
$$

Thus, we see that

$$
L_{n} y(t)+q(t)(1-p[g(t)])^{\beta} G\left(b w_{0}^{*}\left[g(t), t_{2}\right]\right) y^{\beta}[g(t)] \leq 0 \text { for } t \geq t_{3} .
$$

Next, for the case (II), we assume that

$$
p(t) \geq 1, \tau(t)>t \text { and } p(t) \not \equiv 1 \text { eventually. }
$$


We also let

$$
p^{*}(t)=\frac{1}{p\left[h^{-1}(t)\right]}\left(1-\frac{1}{p\left[h^{-1} \circ h^{-1}(t)\right]}\right) \text { for all large } t,
$$

where $h^{-1}$ is the inverse function of $h$. Now, we have

$$
\begin{aligned}
x(t) & =\frac{1}{p\left[h^{-1}(t)\right]}\left(y\left[h^{-1}(t)\right]-x\left[h^{-1}(t)\right]\right) \\
& =\frac{y\left[h^{-1}(t)\right]}{p\left[h^{-1}(t)\right]}-\frac{1}{p\left[h^{-1}(t)\right]}\left(\frac{y\left[h^{-1} \circ h^{-1}(t)\right]}{p\left[h^{-1} \circ h^{-1}(t)\right]}-\frac{x\left[h^{-1} \circ h^{-1}(t)\right]}{p\left[h^{-1} \circ h^{-1}(t)\right]}\right) \\
& \geq \frac{y\left[h^{-1}(t)\right]}{p\left[h^{-1}(t)\right]}-\frac{y\left[h^{-1} \circ h^{-1}(t)\right]}{p\left[h^{-1}(t)\right] p\left[h^{-1} \circ h^{-1}(t)\right]} \\
& \geq \frac{1}{p\left[h^{-1}(t)\right]}\left(1-\frac{1}{p\left[h^{-1} \circ h^{-1}(t)\right]}\right) y\left[h^{-1}(t)\right] \\
& =p^{*}(t) y\left[h^{-1}(t)\right] \text { for } t \geq t_{2} .
\end{aligned}
$$

As before, one can easily obtain

$$
L_{n} y(t)+q(t) f\left(p^{*}[g(t)]\right)^{\beta} G\left(b w_{0}^{*}\left[g(t), t_{2}\right]\right) y^{\beta}\left[h^{-1} \circ g(t)\right] \leq 0 \quad \text { for } \quad t \geq t_{3} .
$$

Now, it is easy to restate results similar to Theorems 3.1, 3.2, 4.1 and 4.2 for equation (5.1) when either (I), or (II) holds. Such formulations are left to the reader.

$\left(\mathrm{I}_{2}\right)$. Suppose $y^{\prime}(t)<0$ for $t \geq t_{1}$. This occurs when we are concerned with bounded solutions of odd order equation (5.1). Here, we can examine the two cases:

$$
(I I I) .\{0 \leq p(t) \leq 1, h(t)>t\} \text { and }(I V) .\{p(t) \geq 1, h(t)<t\} .
$$

One can proceed as above and obtain similar results. Here, we omit the details.

Next, we shall consider a special case of equation (1.1), namely, the equation

$$
\frac{d}{d t}\left(x^{(n-1)}(t)\right)^{\alpha}+q(t) f(x[g(t)])=0 .
$$

In this case one can easily compute the following: For all large $T \geq t_{0}$ and $t \geq s \geq T$, we have

$$
w_{\ell}[t, T]=\frac{(t-T)^{n-2}}{(n-2)(\ell-2) !(n-\ell-1) !}, w_{1}[t, s]=\frac{(t-s)^{n-2}}{(n-2) !} .
$$

Now, it is easy to see that for some $\lambda, 0<\lambda<1$,

$$
\begin{aligned}
w_{\lambda}[t, T] & =\min \left\{w_{1}[t, \lambda t], w_{\ell}[t, T], \quad \ell \in\{1,2, \ldots, n-1\}\right\} \\
& \geq \frac{(\lambda(1-\lambda))^{n-2}}{(n-2) !} t^{n-2} \quad \text { for all large } t
\end{aligned}
$$


(see also, Philos [11]). Inequalities (2.2), (2.3) and (2.4) in Lemma 2.2 take, respectively, the form

$$
\begin{aligned}
x(t) & \geq \frac{\lambda}{(n-1) !} t^{n-1} x^{(n-1)}(t), \\
x^{\prime}(t) & \geq \frac{\lambda}{(n-2) !} t^{n-2} x^{(n-1)}(t)
\end{aligned}
$$

and

$$
x^{\prime}[\lambda t] \geq \frac{(\lambda(1-\lambda))^{n-2}}{(n-2) !} t^{n-2} x^{(n-1)}(t) .
$$

Also, we find

$$
\begin{gathered}
w_{0}[t, s]=\frac{(t-s)^{n-1}}{(n-1) !}, \quad w_{0}^{*}[t, T]=\frac{(t-T)^{n-1}}{(n-1) !}, \\
w_{\ell}[t, T]=\frac{(t-T)^{n-1}}{(n-1)(\ell-1) !(n-\ell-1) !} \text { and } \\
\bar{w}[t, T]=\frac{\lambda}{(n-1) !} t^{n-1} \quad \text { for some } \lambda,
\end{gathered}
$$

$0<\lambda<1$ and all large $t$.

As an example, we restate Theorem 3.1 for equation (5.10). The formulation of other results for equation (5.10) can be done similarly.

Theorem 3.1'. Let the hypotheses of Theorem 3.1 hold with $a_{i}=1, i=$ $1, \ldots, n-1$. Equation (5.10) is oscillatory if equation (3.3) is oscillatory where

$$
p(t)=\rho(t) q(t) G\left(c g^{n-1}(t)\right)+\frac{1}{2}\left(\frac{\rho^{\prime}(t)}{\rho(t) C(t)}\right)^{\prime}-\frac{1}{4 C(t)}\left(\frac{\rho^{\prime}(t)}{\rho(t)}\right)^{2},
$$

$c$ is any positive constant and

$$
\begin{gathered}
\quad \frac{\rho(t)}{\beta \sigma^{\prime}(t)} \sigma^{1-(n-1) \alpha}(t) C(t) \\
=\left\{\begin{array}{l}
c_{1}, c_{1} \text { is any positive constant, when } \beta>\alpha, \\
1, \text { when } \beta=\alpha, \\
c_{2} \sigma^{(n-1)(\beta-\alpha)}(t), c_{2} \text { is any positive constant, when } \beta<\alpha .
\end{array}\right.
\end{gathered}
$$

\section{REFERENCES}

1. R. P. Agarwal and S. R. Grace, On the oscillation of higher order differential equations with deviating arguments. Comput. Math. Appl. 38(1999), 185-199.

2. R. P. Agarwal, S. R. Grace, and D. O'Regan, Oscillation criteria for certain $n$th order differential equations with deviating arguments. J. Math. Anal. Appl. 262(2001), 601-622.

3. R. P. Agarwal, S. R. Grace, and D. O'Regan, Oscillation theory for difference and functional differential equations. Kluwer Academic Publishers, Dordrecht, 2000. 
4. R. P. Agarwal, S. R. Grace, and D. O'Regan, Oscillation theory for second order linear, half-linear, superlinear and sublinear dynamic equations. Kluwer Academic Publishers, Dordrecht, 2002.

5. R. P. Agarwal, S. R. Grace, and D. O'Regan, Oscillation theory for second order dynamic equations. Taylor \& Francis, U.K., 2003.

6. S. R. Grace, Oscillation of even order nonlinear functional differential equations with deviaitng arguments. Funkcial. Ekvac. 32(1989), 265-272.

7. S. R. GRACE, Oscillation theorems of comparison type for neutral nonlinear functional differential equations. Czechoslovak Math. J. 4(1995), 609-626.

8. S. R. GRACE, Oscillation criteria of comparison type for nonlinear functional differential equations. Math. Nachr. 173(1995), 177-192.

9. M. K. KWONG and J. S. W. Wong, Linearization of second order nonlinear oscillation theorems. Trans. Amer. Math. Soc. 279(1983), 705-722.

10. W. E. Mahfoud, Remarks on some oscillation theorems for $n$th order differential equations with a retarded argument. J. Math. Anal. Appl. 62(1978), 60-80.

11. Ch. G. Philos, Some comparison criteria in oscillation theory. J. Austral. Math. Soc. Ser. A 36(1984), 176-186.

(Received 8.08.2003)

Authors' addresses:

Ravi P. Agarwal

Department of Mathematical Sciences

Florida Institute of Technology

Melbourne, FL 32901

U.S.A.

E-mail: agarwal@fit.edu

Said R. Grace

Department of Engineering Mathematics

Faculty of Engineering, Cairo University

Orman, Giza 12221

Egypt

E-mail: srgrace@alpha1-eng.cairo.eun.eg

Donal O'Regan

Department of Mathematics

National University of Ireland, Galway

Ireland

E-mail: donal.oregan@nuigalway.ie 